\title{
Influência das variáveis ambientais na fauna acompanhante na pesca da manjuba Anchoa tricolor (Agassiz) (Actinopterygii, Engraulidae) na Baía de Sepetiba, Rio de Janeiro
}

\author{
Márcio de Araújo Silva \& Francisco Gerson Araújo
}

Laboratório de Ecologia de Peixes, Universidade federal Rural do Rio de Janeiro. Antiga Rodovia Rio-São Paulo, Km 47, 23851-970 Seropédica, Rio de Janeiro, Brasil.

\begin{abstract}
Influences of environmental parameters on the accompanying fauna in the anchovy fisheries of Anchoa tricolor (Agassiz) (Actinopterygii, Engraulidae) in the Sepetiba Bay, Rio de Janeiro. Anchoa tricolor (Agassiz, 1829) is the only anchovy of commercial importance in Sepetiba Bay, supporting an intensive commercial fishery by small sized seine boats provide of hydroacustics sounder detecting device. This study analyses the accompanying fauna in the $A$. tricolor fishery, and the influence of environmental parameters in fish composition. Seven samples were taken with a seine net between March and September 1999, capturing 114,430 fish, comprising 17 species and 11 families. Engraulidae and Clupeidae were the most abundant families, accounting to $94.2 \%$ of the total number of fish. A. tricolor was the most abundant species contributing to more than one half of the total numerical abundance, followed in decreasing order by Anchoa januaria (Steindachner, 1879), Sardinella brasiliensis (Steindachner, 1789) and Harengula clupeola (Cuvier, 1829). A. tricolor showed a highly significant positive correlation with salinity, transparency and depth. A. januaria showed the opposite, being inversely correlated to these parameters and directly correlated to temperature. Clupeidae, on the other hand, seems to show a wider distribution in relations to these environmental parameters.
\end{abstract}

KEY WORDS. Anchoa tricolor, Engraulidae, pelagic fishes, Sepetiba Bay.

Apesar de Anchoviella lepidentostole (Fowler, 1911) ser considerada como a única espécie de Engraulidae de importância econômica na região sudeste do Brasil (Figueiredo \& Menezes 1978, Paiva Filho et al. 1986, 1990), Anchoa tricolor (Agassiz, 1829) é a única manjuba de importância comercial na Baía de Sepetiba, distribui-se na zona pelágica costeira, concentrandose em grandes cardumes em regiões semi-abertas, como baías e estuários, o que faz com que seja pescada em grandes quantidades (Coto et al. 1988, Cowan Jr. \& Shaw 1991, MacGregor \& Houde 1996). Exemplares dessa família desempenham importante papel na cadeia alimentar dos oceanos, servindo de forragem a muitas espécies de peixes e aves marinhas (Hildebrand 1963).

A pesca da manjuba é praticada por embarcações de pequeno porte (comprimento $<15 \mathrm{~m}$ ) e a localização destes peixes é feita com o auxílio de ecossonda tipo RV400.

No presente trabalho, é analisada a composição da fauna acompanhante na pesca de $A$. tricolor, bem como a influência das variáveis ambientais como: temperatura, salinidade, transparência e profundidade no êxito desta pesca e na estruturação da ictiofauna da Baía de Sepetiba.

\section{MATERIAL E MÉTODOS}

A Baía de Sepetiba localiza-se ao Sul do Estado do Rio de Janeiro entre as latitudes $22^{\circ} 54^{\prime}$ e $23^{\circ} 04^{\prime} \mathrm{S}$ e as longitudes $43^{\circ} 34^{\prime}$ e $44^{\circ} 10^{\prime} \mathrm{W}$ (Fig. 1). A baía apresenta forma alongada e limita-se ao Norte e ao Leste pelo continente, ao Sul pela Restinga da Marambaia e a Oeste pela Baía da Ilha Grande. Seu comprimento é de $43 \mathrm{~km}$ no sentido Leste-Oeste e sua maior largura de $17 \mathrm{~km}$ no sentido Norte-Sul, sendo o perímetro de aproximadamente $123 \mathrm{~km}$. A bacia hidrográfica apresenta área aproximada de $3500 \mathrm{~km}^{2}$ (ArAújo et al. 1998).

A margem continental em razão da existência da Restinga e de algumas ilhas caracteriza-se por ser uma zona de poucos batimentos, apresentando, portanto, um substrato principalmente lodoso, com formações de silte, argila e com poucas áreas de areia e cascalho. As praias são do tipo arenosas, lodopedregosas, de cascalhos e ainda, a combinação destes vários tipos (ARAújo et al. 1997).

Foram realizadas sete amostragens entre março e setembro de 1999, usando barco tipo traineira, com $12 \mathrm{~m}$ de comprimento, equipado com ecossonda e provido com rede 
de cerco $($ comprimento $=240 \mathrm{~m}$, altura $=27 \mathrm{~m}$, malha da parte superior $=8 \mathrm{~mm}$, malha da parte inferior $=10 \mathrm{~mm}$ ).

As amostragens 1 e 2 foram realizadas em março, a 3 em julho, 4 e 5 em agosto, 6 e 7 em setembro (Fig. 1). Em cada amostragem foram registrados os fatores ambientais de temperatura da água, salinidade, transparência e profundidade. A temperatura foi medida através do termômetro com coluna de mercúrio com precisão $0,1^{\circ} \mathrm{C}$ e a salinidade, com salinômetro óptico, com precisão de 0,5 . A transparência (em centímetros) foi obtida através de um disco de Secchi, e a profundidade (em metros) foi registrada com ecossonda.

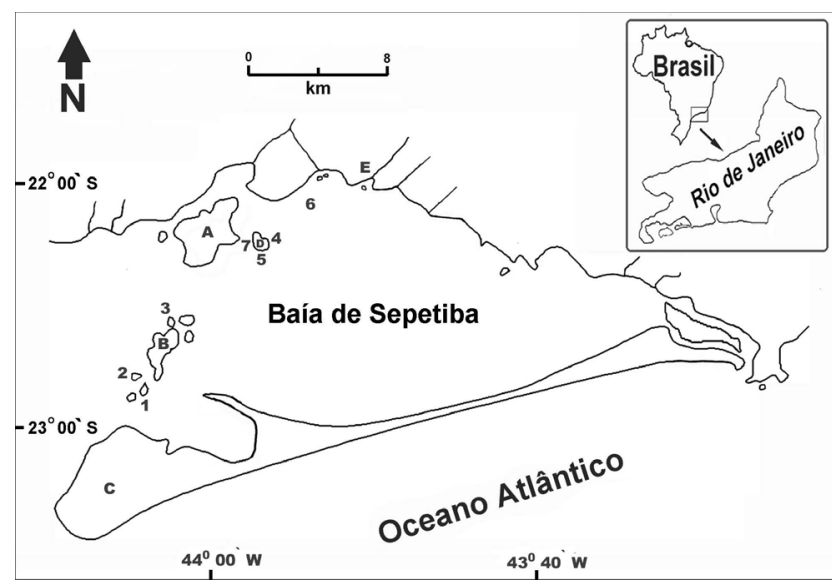

Figura 1. Baía de Sepetiba com indicação dos locais de amostragem (1 a 7), das principais ilhas - (A) Itacuruçá, (B) Jaguanum, (C) Marambaia, (D) Martins - e (E) Rio Guandú.

A operação de pesca foi monitorada por ecossonda modelo Royal RV400 que também foi utilizada para detecção da presença dos cardumes sob a embarcação. As amostragens foram feitas de preferência nas proximidades das ilhas Itacuruça, Jaguanum e Martins (Fig. 1). Após a detecção do cardume, eram realizados os lançamentos da rede com o auxílio de um bote utilizado para ancorar a rede, enquanto a traineira efetuava o cerco do cardume, fechando rede. O recolhimento da rede é feito com o fechamento da tralha inferior.

De cada amostragem (cerco), foi tomado o peso total dos peixes, separado por espécies. Para os indivíduos com uma captura relativamente pequena, todos os exemplares foram levados para a análise no laboratório juntamente com uma sub-amostra (aproximadamente $1 / 20$ da captura) dos indivíduos que apresentaram grande abundancia. No laboratório foi feita a pesagem dos indivíduos, e, por extrapolação, no caso dos indivíduos mais abundantes, estimadas a contribuição numérica, das espécies na captura. Os dados de abundância dos peixes foram transformados usando o logaritmo decimal, visando à normalidade e homocedasticidade, e também a diminuição da influência das espécies muito abundantes. A correlação entre $A$. tricolor e as demais espécies de peixes, bem como entre as variáveis ambientais e as espécies mais abundantes, foi determinada através do índice de classificação $r$ Spearman. As espécies mais abundantes foram definidas como aquelas que contribuíram acima de $5 \%$ do número total de peixes capturados. Também foi utilizada a análise multivariada de agrupamento para determinação de eventuais grupos de espécies ditados por semelhantes padrões de ocorrência e separações dos locais de amostragens. Nessa análise, foram utilizados a distância Euclidiana e o método de agrupamento WPGA (weighted pair group average).

\section{RESULTADOS}

A salinidade nos locais amostrados variou de 18 a 30 , enquanto que a temperatura foi de 22 a $28,7^{\circ} \mathrm{C}$. A transparência variou de 70 a $205 \mathrm{~cm}$, e a profundidade, de 5 a $10 \mathrm{~m}$.

A análise de agrupamento feita entre os locais de amostragens determinou três grupos ao nível de 3 de distância Euclidiana; um grupo compreendido pelas amostragens 1 e 2 (A), outro pelas amostragens 3, 4, 5, 7 (B), sendo a amostragem 6 diferente das demais (C) (Fig. 2). A análise das variáveis ambientais dentro de cada grupo apresentou o grupo A como o de maior valor de temperatura, salinidade e profundidade. $\mathrm{O}$ grupo $B$ apresentou maiores valores de transparência, enquanto a amostragem 6 (evidenciada como C) apresentou menores valores de salinidade, transparência e profundidade (Fig. 3).

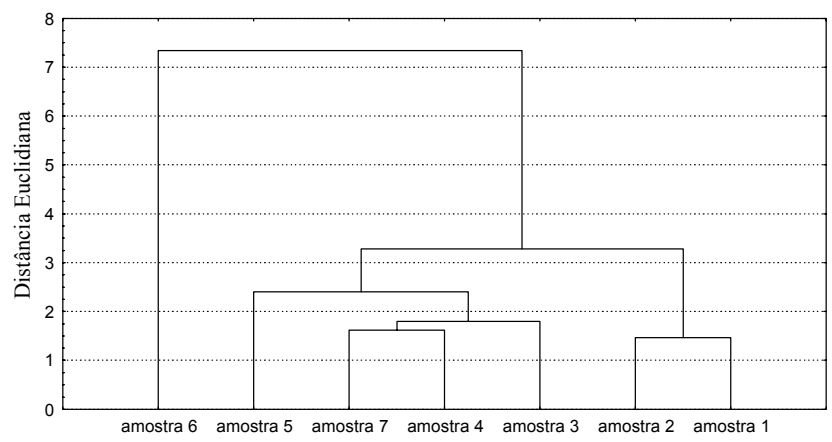

Figura 2. Dendrograma mostrando o agrupamento das amostragens.

Foi capturado um total de 114.430 peixes, compreendendo 17 espécies, em 11 famílias, com média de cinco espécies por amostragem (cerco). As famílias Engraulidae e Clupeidae corresponderam a $94,2 \%$ do número total de peixes capturados (Tab. I). Anchoa tricolor foi a espécie mais abundante com mais da metade do total capturado, seguida em ordem decrescente de abundância por Anchoa januaria (Steindachner, 1879), Sardinella brasiliensis (Steindachner, 1789) e Harengula clupeola (Cuvier, 1829).

Anchoa tricolor ocorreu em todas as amostragens com exceção da amostragem 6 , quando foi feito o único registro de A. januaria, com esta última espécie tendo sido o peixe de maior abundância da amostra. S. brasiliensis ocorreu em elevada abundância nas amostragens 1, 2 (grupo A) e 6 (grupo C), enquanto H. clupeola, nas amostragens 3, 4 e 7 (grupo B), figurando como a segunda espécie de maior abundância, após $A$. tricolor (Fig. 4). 

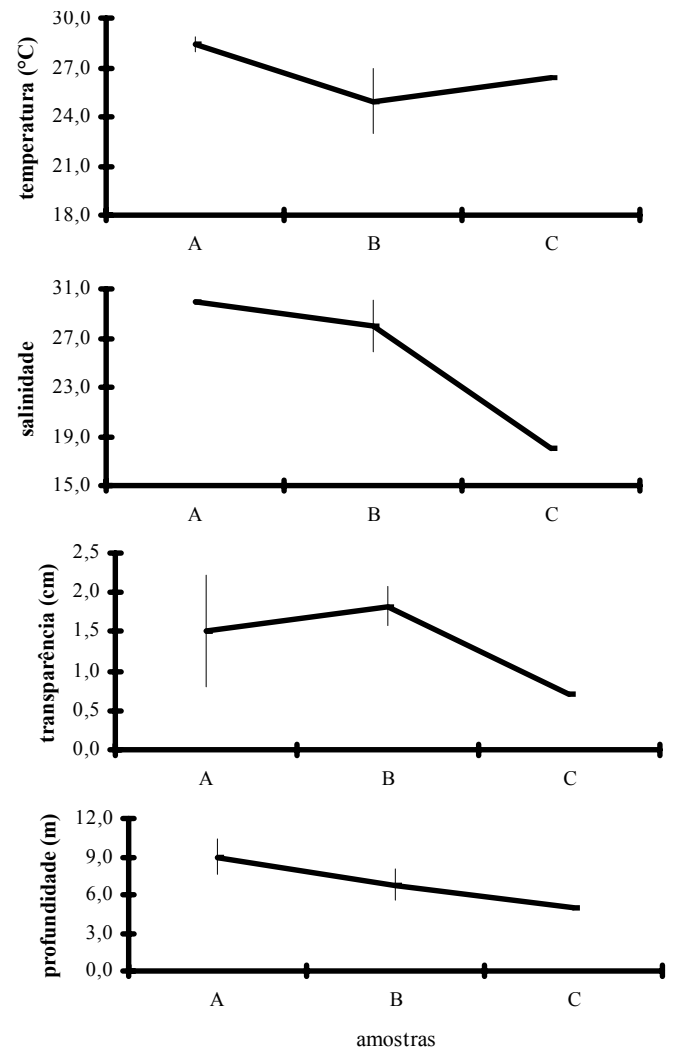

Figura 3. Médias e desvios padrões dos fatores ambientais nos locais de amostragem agrupados pelo cluster.

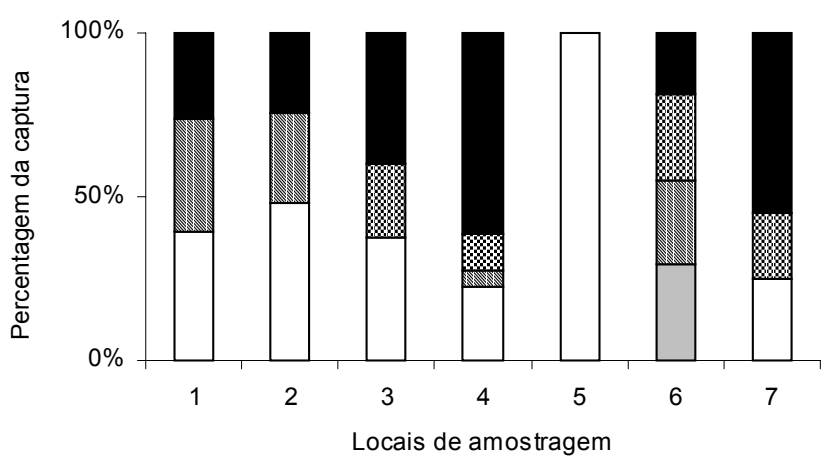

$\square$ A. tricolor $\square$ A. januaria $\mathbf{S}$. brasiliensis $\mathbf{H}$. clupeola $\square$ Outros

Figura 4. Abundância relativa das espécies mais freqüentes.

Anchoa tricolor apresentou uma correlação positiva altamente significante com a salinidade e com a transparência, e significante com a profundidade (Tab. II); enquanto que $A$. januaria apresentou o inverso desta situação, com uma correlação negativa altamente significante com a salinidade, transparência, profundidade e uma correlação positiva altamente
Tabela I. Abundância das espécies capturadas nos arrastos de meia água na Baía de Sepetiba, Rio de Janeiro.

\begin{tabular}{|c|c|c|c|}
\hline Nome Científico & Nome Comum & Total & $\%$ \\
\hline \multicolumn{4}{|l|}{ Engraulidae } \\
\hline Anchoa tricolor & Manjuba-verdadeira & 63867 & 55,813 \\
\hline Anchoa januaria & Manjuba-prata & 23529 & 20,562 \\
\hline Cetengraulis edentulus & Sardinha-boca torta & 689 & 0,602 \\
\hline \multicolumn{4}{|l|}{ Clupeidae } \\
\hline Sardinella brasiliensis & Sardinha-verdadeira & 11201 & 9,789 \\
\hline Harengula clupeola & Sardinha-laje & 10021 & 8,757 \\
\hline \multicolumn{4}{|l|}{ Carangidae } \\
\hline Chloroscombrus chrysurus & Palombeta & 4004 & 3,499 \\
\hline Oligoplites palometa & Guaivira & 6 & 0,005 \\
\hline \multicolumn{4}{|l|}{ Gerreidae } \\
\hline Eucinostomus gula & Carapicu & 805 & 0,703 \\
\hline Diapterus rhombeus & Carapeba & 195 & 0,170 \\
\hline \multicolumn{4}{|l|}{ Sciaenidae } \\
\hline Ctenosciaena gracillicirrhus & Porrudo & 84 & 0,073 \\
\hline Micropogonias furnieri & Corvina & 12 & 0,010 \\
\hline \multicolumn{4}{|l|}{ Tetraodontidae } \\
\hline Sphoeroides testudineus & Baiacu & 6 & 0,005 \\
\hline \multicolumn{4}{|l|}{ Triglidae } \\
\hline Prionotus punctatus & Cabrinha & 5 & 0,004 \\
\hline \multicolumn{4}{|l|}{ Haemulidae } \\
\hline Orthopristis ruber & Cocoroca & 3 & 0,003 \\
\hline \multicolumn{4}{|l|}{ Sparidae } \\
\hline Archosargus rhomboidalis & Salema & 1 & 0,001 \\
\hline \multicolumn{4}{|l|}{ Trichiuridae } \\
\hline Trichiurus lepturus & Espada & 1 & 0,001 \\
\hline \multicolumn{4}{|l|}{ Ariidae } \\
\hline Sciadeichthys luniscutis & Bagre & 1 & 0,001 \\
\hline
\end{tabular}

significativa com a temperatura. As espécies da família Clupeidae apresentaram uma correlação positiva significante com a temperatura.

A análise de agrupamento das espécies resultou em três grupos ao nível de dois de distância Euclidiana; um compreendido por Ctenosciaena gracillicirrhus (Metzelaar, 1919) e Eucinostomus gula (Cuvier, 1830), outro por A. januaria e C. edentulus (Cuvier, 1828) e um terceiro pelas demais espécies, com exceção de A. tricolor, H. clupeola, Chloroscombrus chrysurus (Linnaeus, 1766) e $S$. brasiliensis que se apresentaram isoladas (Fig. 5). 


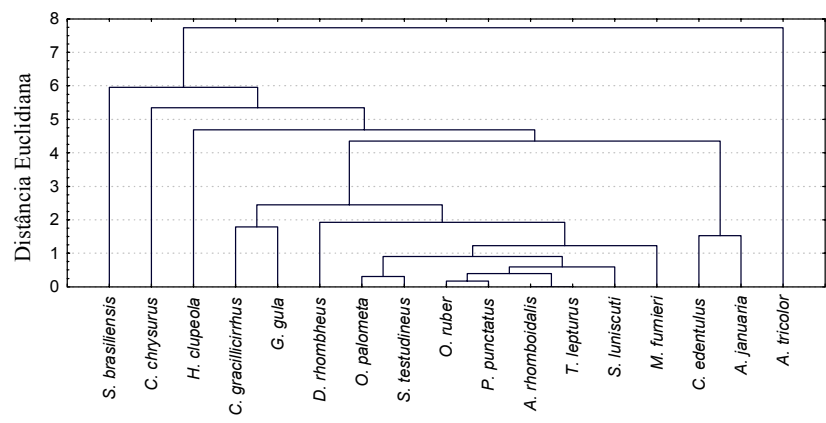

Figura 5. Dendrograma mostrando o agrupamento das espécies.

Tabela II. Valores de r (Spearman) e nível de significância da correlação entre a família Engraulidae e Clupeidae com os fatores ambientais.

\begin{tabular}{|c|c|c|c|c|}
\hline \multirow{2}{*}{ Espécies } & \multicolumn{4}{|c|}{ Variáveis ambientais } \\
\hline & Salinidade & Temperatura & Transparência & Profundidade \\
\hline A. tricolor & 0,78 ** & $-0,05 \mathrm{NS}$ & 0,95 ** & $0,44^{*}$ \\
\hline A. januaria & $-0,73 * *$ & 0,52 ** & $-0,73 * *$ & $-0,58 * *$ \\
\hline S. brasiliensis & $-0,45 *$ & 0,87 ** & $-0,41$ * & $-0,22$ NS \\
\hline H. clupeola & $-0,24$ NS & 0,37 * & $-0,28 \mathrm{NS}$ & $-0,33 \mathrm{NS}$ \\
\hline
\end{tabular}

(*) Significante ao nível de 95\%, (**) significante ao nível de 99\%, (NS) não significativo.

Anchoa tricolor apresentou correlação positiva significante com a maioria das espécies, porém foi inversamente correlacionada com A. januaria e C. edentulus, e em menor intensidade, com $S$. brasiliensis (Tab. III).

\section{DISCUSSÃO}

A grande abundância de $A$. tricolor mostra que a arte de pesca utilizada é extremamente seletiva, objetivando capturar quase que exclusivamente esta espécie. A pesca de meia água, quando realizada com barcos providos de rede de arrasto, necessita de intrumentos hidroacústicos, visando também controlar a altura da rede para as necessidades de amostragem em toda a coluna d'água (MacLennan \& Simmonds 1992, Habiaga et al. 1997). No caso do presente trabalho, em que a pesca é realizada com rede de cerco, operando em áreas pouco profundas, como as regiões de baías, a necessidade do ecossonda para monitorar a altura da rede torna-se desnecessária, sendo este aparelho utilizado tão somente para determinar a presença dos cardumes. Nestes casos de baías, a rede de cerco é uma opção de arte de pesca que apresenta maior produtividade em relação à rede de arrasto de meia água, porém seu uso é dificultado em zonas de baixa profundidade $(<5 \mathrm{~m})$.

Whitehead et al. (1988) reportam que os representantes das famílias Engraulidae e Clupeidae apresentam várias semelhanças comportamentais, constituindo cardumes pelágicos de caráter transitório ou permanente dentro dos estuários. Na Baía
Tabela III. Valor de r (Spearman) e nível de significância da correlação entre Anchoa tricolor e as demais espécies capturadas.

\begin{tabular}{lc}
\hline \multicolumn{1}{c}{ Espécies } & Valor de R \\
\hline A. tricolor Vs. A. januaria & $-0,72 * *$ \\
A. tricolor Vs. C. edentulus & $-0,72 * *$ \\
A. tricolor Vs. C. chrysurus & $0,65^{* *}$ \\
A. tricolor Vs. S. brasiliensis & $-0,38^{*}$ \\
A. tricolor Vs. M. furnieri & $0,59 *$ \\
A. tricolor Vs. S. luniscuti & $0,03 \mathrm{NS}$ \\
A. tricolor Vs. H. clupeola & $-0,22 \mathrm{NS}$ \\
A. tricolor Vs. E. gula & $0,88 * *$ \\
A. tricolor Vs. D. rhombheus & $0,61 * *$ \\
A. tricolor Vs. T. lepturus & $0,61 * *$ \\
A. tricolor Vs. S. testudineus & $0,78 * *$ \\
A. tricolor Vs. A. rhomboidalis & $0,61 * *$ \\
A. tricolor Vs. P. punctatus & $0,61 * *$ \\
A. tricolor Vs. O. ruber & $0,61 * *$ \\
A. tricolor Vs. C. gracillicirrhus & $0,78 * *$ \\
A. tricolor Vs. O. palometa & $0,47^{*}$ \\
\hline
\end{tabular}

(*) Significante ao nível de 95\%, $\left(^{* *}\right)$ significante ao nível de 99\%, (NS) não significativo.

de Sepetiba isso pode ser bem evidenciado, já que 94,2\% do pescado capturado pertenciam a estas duas famílias.

Através da análise de agrupamento, pode ser observada a formação de determinados grupos de peixes associados a determinadas características ambientais. As variáveis ambientais como a salinidade, profundidade e transparência da água, foram determinantes para a separação de $A$. tricolor de $A$. januaria, sendo $A$. tricolor mais abundante em salinidade mais elevadas, enquanto que $A$. januaria foi mais abundante nas salinidades mais baixas. Uma indicação provável é a concentração dos barcos de pesca, que são direcionados para captura da espécie de valor comercial (A. tricolor) em áreas distantes da influência dos rios, já que estes locais apresentaram menores gradientes de salinidade e elevada abundância de $A$. januaria. Com relação à transparência e à profundidade, pode ser ressaltado que os locais que apresentaram menores valores destas variáveis ambientais foram os locais mais internos da baía, e de maior influência dos rios, evidenciando que, $A$. januaria é uma espécie mais associada ao interior de baías, onde a influência da água de drenagem continental é maior, enquanto $A$. tricolor ocorre mais associada a regiões costeiras semi abertas, com maior influência de águas marinhas. CarvalHo (1950) relatou que as formas jovens de $A$. tricolor, durante o seu crescimento, foram encontrados na Baía de Guanabara, enquanto que os adultos, em alto mar, aproximando-se da costa na época de reprodução. Com relação a A. januaria, Silva \& AraúJo (1999) relataram que esta espécie foi mais abundante na zona mais interna. 
Os componentes da família Clupeidae, apesar de associados à ocorrência de $A$. tricolor, parecem apresentar uma mais ampla distribuição na baía, tendo inclusive sido abundantes em salinidade com gradiente relativamente baixo, juntamente com A. januaria.

As correlações positivas de $A$. tricolor com a maioria das demais espécies capturadas pode ser atribuída ao fato desta pesca ser direcionada basicamente para essa espécie.

\section{REFERÊNCIAS BIBLIOGRÁFICAS}

Araújo, F.G.; A.G. Cruz-Filho; M.C.C. Azevedo \& A.C.A. Santos. 1998. Estrutura da comunidade de peixes demersais da Baía de Sepetiba, RJ. Revista Brasileira de Biologia, São Carlos, 58 (3): 417-430.

Araújo, F.G.; A.G. Cruz-Filho; M.C.C. Azevedo; A.C.A. Santos \& L.A.M. FERNANDES. 1997. Estrutura da comunidade de peixes jovens da margem continental da Baía de Sepetiba, RJ. Acta Biológica Leolpoldensia, São Leopoldo, 19 (1): 61-83.

Carvalho, J.P. 1950. Engraulídeos brasileiros do gênero Anchoa. Boletim do Instituto Paulista de Oceanografia, São Paulo, 1: 43-70.

Coto, C.F.; A.O. Luna; A.L. Calvo; F.Z. García. 1988. Abundancia de algunas especies de Anchoas en la laguna de Términos (México), estimada a través de la captura de huevos. Annal del Institute de Ciências del Mar y Limnologia da Universidad Autónoma de México, México, 15 (1): 125134.

CoWAN JR., J.H.; R.F. SHAw. 1991. Ichthyoplankton off West Louisiana in Winter 1981-1982 and its Relatioship with Zooplankton Biomass. Contributions in Marine Science, Ijmuiden, 32.

Figueiredo, J.L. \& N.A. Menezes. 1978. Manual de peixes marinhos do sudeste do Brasil. II. Teleostei (1). São Paulo,
Museu de Zoologia, Universidade de São Paulo, 110p.

Habiaga, R.; C. Soares; L. Madureira \& D. Eliseire JR. 1997. Rede de meia água: operação e eficiência como amostrador em cruzeiros de ecointegração. Atlântica, Rio Grande, 19: 145-159.

Hildebrand, S.F. 1963. Family Engraulidae. In Fishes of the Western North Atlantic. Memoirs of Sears Fundation for Marine Research, New Haven, 1 (3): 152-249.

MacLennan, D.N. \& E.J. Simmonds. 1992. Fisheries Acoustics. London, Chapman \& Hall, Fish and Fisheries Series 5, 325p.

MACGREgor, J.M.; E.D. Houde. 1996. Onshore-Offshore pattern and variability in distribution and abundance of bay anchovy Anchoa mitchilli eggs and larvae in Cheasapeake Bay. Marine Ecology Progress Series, Nordbunte, 138: 1525.

Paiva-Filho, A.M.; M.L. Zani Teixeira. \& P.K. Kinara. 1986. Contribuição ao conhecimento da Biologia da manjuba, Anchoviella lepidentostole (Fowler, 1911), no estuário de São Vicente, SP (Osteichthyes, Engraulidae). Boletim do Instituto Oceanográfico, São Paulo, 34: 71-77.

Paiva-Filho, A.M.; R. Giannini \& F.B.R. Neto. 1990. A pesca da manjuba Anchoviella lepidentostole (Engraulidae) no estuário de São Vicente, SP. Relatório Interno do Instituto Oceanográfico, São Paulo, 29: 1-6.

Silva, M.A. \& F.G. AraúJo. 1999. Influência dos fatores ambientais na estrutura de populações de manjubas (ClupeiformesEngraulidae) na Baía de Sepetiba, RJ. Acta Biológica Leolpoldensia, São Leopoldo, 21 (2): 229-240.

Whitehead, P. J. P.; G. J. Nelson \& T. Wongratana. 1988. FAO species catalogue. Vol. 7. Clupeoid fishes of the world (Suborder Clupeoidei). An annotated and illustrated catalogue of the herrings, sardines, pilchards, sprats, shads, anchovies and wolf-herrings. Part 2 - Engraulididae. Rome, FAO Fish Synopsis, 7, 579p.

Recebido em 29.VII.2002; aceito em 21.IV.2003. 